\title{
Upregulation of metastasis-associated gene 2 promotes cell proliferation and invasion in nasopharyngeal carcinoma
}

This article was published in the following Dove Press journal:

OncoTargets and Therapy

18 March 2016

Number of times this article has been viewed

\section{Minhua $\mathrm{Wu}^{1,2, *}$ \\ Xiaoxia $\mathrm{Ye}^{2, *}$ \\ Xubin Deng,* \\ Yanxia $\mathrm{Wu}^{4}$ \\ Xiaofang $\mathrm{Li}^{4}$ \\ Lin Zhang'}

'Department of Histology and Embryology, Southern Medical University, Guangzhou, ${ }^{2}$ Department of Histology and Embryology, Guangdong Medical University, Zhanjiang, ${ }^{3}$ Affiliated Cancer Hospital of Guangzhou Medical University, Cancer Center of Guangzhou Medical University, Guangzhou, ${ }^{4}$ Pathological Diagnosis and Research Center, Affiliated Hospital of Guangdong Medical University, Zhanjiang,

People's Republic of China

*These authors contributed equally to this work
Correspondence: Lin Zhang Department of Histology and Embryology, Southern Medical University, 1838 North Guangzhou Avenue, Guangzhou 5I05I5, People's Republic of China

Tel +8620I648205

Email zlilyzh@I26.com
Aims: Metastasis-associated gene $2(M T A 2)$ is reported to play an important role in tumor progression, but little is known about the role of MTA2 in nasopharyngeal carcinoma (NPC). The aim of the study was to explore the expression and function of MTA2 in NPC.

Methods: Expression of MTA2 in NPC tissues and cell lines was detected by immunohistochemistry and Western blotting. Relationship between MTA2 expression and clinicopathological features was analyzed. Stable MTA2-overexpressing and MTA2-siliencing NPC cells were established by transfection with plasmids encoding MTA2 cDNA and lentivirus-mediated short hairpin RNA, respectively. Cell viability was determined by Cell Counting Kit- 8 and colony formation assay. Cell migration ability was evaluated by wound healing and transwell invasion assay. The impact of MTA2 knockdown on growth and metastasis of CNE2 cells in vivo was determined by nude mouse xenograft models. Expression of several Akt pathway proteins was detected by Western blotting.

Results: MTA2 was upregulated in NPC tissues and three NPC cell lines detected (CNE1, CNE2, and HNE1). MTA2 expression was related to clinical stage and lymph node metastasis of patients with NPC. MTA2 upregulation promoted proliferation and invasion of CNE1 cells, while MTA2 depletion had opposite effects on CNE2 cells. Moreover, MTA2 depletion suppressed growth and metastasis of CNE2 cells in vivo. MTA2 overexpression activated Akt and upregulated the expression of matrix metalloproteinase 7 and cyclin D1.

Conclusion: We conclude that MTA2 acts as an oncogene in tumorigenesis of NPC. MTA2 may be a potential target for gene therapy in NPC.

Keywords: MTA2, nasopharyngeal carcinoma, proliferation, invasion, Akt pathway

\section{Introduction}

Nasopharyngeal carcinoma (NPC) is one of the most prevalent cancers in Southeast Asia and South China. Although the treatment of NPC has improved greatly in recent years with the development of radiotherapy, the 5-year survival rate of patients with NPC is still $<60 \%$. Approximately 20,000 people die of NPC every year in the People's Republic of China. ${ }^{1,2}$ The main cause of treatment failure and patient's mortality is tumor metastasis, but the molecular mechanism of tumorigenesis and metastasis of NPC are still elusive. It will be of great clinical value to find out new biomarkers involved in the progression of NPC.

Metastasis-associated gene (MTA) family is a novel gene family. ${ }^{3}$ MTA1, MTA2, and MTA3 are the main members of this family. They are components of nucleosome remodeling and deacetylation complex. ${ }^{4}$ As a part of the nucleosome remodeling and deacetylation complex, MTAs are proved to modulate gene transcription by affecting 
chromatin remodeling. ${ }^{5,6}$ MTA2, as a member of $M T A$ gene family, is highly expressed in human osteosarcoma, ${ }^{7}$ colorectal cancer, ${ }^{8}$ ovarian cancer, ${ }^{9}$ lung cancer, ${ }^{10}$ and several other human tumor tissues. High MTA2 expression is positively related to metastasis and poor prognosis in esophageal carcinoma $^{11}$ and pancreatic carcinoma. ${ }^{12}$

Expression of MTA2 affects tumor cell growth, metastasis, apoptosis, and cell skeleton. ${ }^{13,14}$ Silencing of MTA2 reverses the malignant phenotypes of glioma, ${ }^{15}$ gastric cancer, ${ }^{16}$ and breast cancer ${ }^{17}$ cells. These studies strongly suggest a correlation between tumor development and MTA2 expression. However, to the best of our knowledge, the expression pattern and function of MTA2 in NPC have not been reported before.

\section{Materials and methods Patients and samples}

Samples of 107 NPC tissues and 28 noncancerous nasopharyngeal epithelium tissues were obtained from biopsy of patients from the Affiliated Hospital of Guangdong Medical University (Zhanjiang, People's Republic of China) between 2010 and 2011. None of the patients with NPC received radiotherapy or chemotherapy before biopsy. The clinicopathological information was obtained from medical records. Staging of cancer was in accordance with the seventh American Joint Committee on Cancer and the International Union for Cancer Control cancer staging guidelines issued in 2010. The study was approved by the Medical Ethics Committee of the Affiliated Hospital of Guangdong Medical University. Written informed consent was obtained from each patient. Patients consisted of 71 males and 36 females with an average age of 48 years (range: $18-75$ years).

\section{Immunohistochemistry}

Sections from patients were deparaffinized with xylene and rehydrated with graded alcohol. For antigen retrieval, sections were immersed in citrate buffer $(\mathrm{pH}=6.0)$ and microwaved for 5 minutes. To eliminate intrinsic peroxidase activity, slides were incubated with $3 \% \mathrm{H}_{2} \mathrm{O}_{2}$ for 8 minutes. Slides were incubated overnight at $4^{\circ} \mathrm{C}$ with a goat antibody against MTA2 (dilution, 1:300; Santa Cruz Biotechnology Inc., Dallas, TX, USA). After washing with phosphatebuffered saline (PBS), sections were detected with a Polink-2 Plus Polymer HRP Kit (GBI, Mukilteo, WA, USA) according to the manufacturer's instructions. Signals were visualized with 3,3'-diaminobenzidine tetrahydrochloride for 2 minutes and slightly counterstained with hematoxylin. The primary antibody was replaced with PBS as a negative control. The slides were evaluated independently by two investigators who were blinded to the patient's clinicopathological information. The degree of immunohistochemical staining was assessed by the positive cell proportion and intensity of staining. ${ }^{18}$ The percentage of positivity was scored as follows: $0(<5 \%)$, $1(5 \%-25 \%), 2(26 \%-50 \%)$, and $3(>50 \%)$. The staining intensity was scored as follows: 0 (no), 1 (light yellow), 2 (yellow), and 3 (brown yellow). The final score was calculated by multiplying the proportion score and intensity score. Slides with a final score of $\geq 4$ were defined as positive; otherwise, they were defined as negative.

\section{Cell culture}

Human NPC cell lines CNE1, CNE2, and HNE1 and human immortalized nasopharyngeal epithelium cell line NP6 $6{ }^{19}$ were obtained from the Cancer Research Institute of Guangdong Medical University and Southern Medical University, Guangdong, People's Republic of China. The use of these cell lines were approved by the institutional review boards of both of the aforementioned schools. CNE1, CNE2, and HNE1 cells were cultured in Dulbecco's Modified Eagle's Medium (DMEM) supplemented with $10 \%$ fetal bovine serum (FBS; Thermo Fisher Scientific, Waltham, MA, USA) at $37^{\circ} \mathrm{C}$ with $5 \% \mathrm{CO}_{2}$ and a humidified atmosphere. NP69 cells were grown in keratinocyte serum-free medium (Thermo Fisher Scientific).

\section{Quantitative reverse transcription- polymerase chain reaction analysis}

Quantitative real-time reverse transcription-polymerase chain reaction (qRT-PCR) assay was performed as previously described. ${ }^{20}$ Briefly, RNAiso Plus reagent (Takara, Dalian, People's Republic of China) was used to extract total RNA of cells. The cDNA was obtained from messenger RNA with a Primescript ${ }^{\mathrm{TM}}$ RT reagent kit (Takara). A SYBR Green PCR kit (Takara) was used in the amplification processes by a Light Cycler system (Hoffman-La Roche Ltd, Basel, Switzerland). The primers used for amplification of MTA2 and $\beta$-actin were as follows: sense 5'-GATGAGATGGAGGAATGGTCAG-3', antisense 5'-ACCTGTTTCAGTTTGCTGTCTG-3' (for MTA2); sense 5'-TGACGTGGACATCCGCAAAG-3', and antisense 5'-CTGGAAGGTGGACAGCGAGG-3' (for $\beta$-actin). The cycling program was as follows: denaturation at $95^{\circ} \mathrm{C}$ for 30 seconds, followed by 40 cycles of denaturing at $95^{\circ} \mathrm{C}$ for 5 seconds, annealing at $60^{\circ} \mathrm{C}$ for 30 seconds. The qRT-PCR result was analyzed with the $2^{-\Delta \Delta \mathrm{Ct}}$ method. ${ }^{21}$ $\beta$-actin was used as an internal control.

\section{Western blot analysis}

Western blot experiment was performed as previously described. ${ }^{22}$ Briefly, cells or tumor tissues were lysed with RIPA 
reagent (Beyotime, Shanghai, People's Republic of China). The concentration of protein was determined by bicinchoninic acid method. An equal amount of protein in each lane was subjected to $10 \%$ sodium dodecyl sulfate-polyacrylamide gel electrophoresis and transferred to polyvinylidene fluoride membranes (EMD Millipore, Billerica, MA, USA). After blocking for 1 hour with $5 \%$ nonfat milk, the polyvinylidene fluoride membranes were incubated with MTA2 and $\beta$-actin polyclonal antibody (dilution, 1:1,000; Santa Cruz Biotechnology Inc.), Akt and pAkt (Ser473) monoclonal antibody (dilution, 1:2,000; Cell Signaling Technology, USA), cyclin D1 monoclonal antibody (dilution, 1:1,500; Cell Signaling Technology), hypoxiainducible factor $1 \alpha$ monoclonal antibody (dilution, 1:300; Boster Biotechnology, Wuhan, People's Republic of China), and matrix metalloproteinase 7 (MMP7) polyclonal antibody (dilution, 1:1,000; Abcam, Cambridge, UK). After incubation with primary antibody at $4^{\circ} \mathrm{C}$ overnight, the membranes were incubated with secondary antibody (dilution, 1:5,000; Santa Cruz Biotechnology Inc.) at room temperature for 1 hour. Signals were visualized with the enhanced chemiluminescence detection system (Pierce, Rockford, IL, USA). The intensity of protein bands was quantified using Quantity One software (Bio-Rad Laboratories Inc., Hercules, CA, USA). The protein levels were normalized to those of $\beta$-actin.

\section{Establishment of stable MTA2- overexpressing CNEI cell}

The pcDNA3.1 plasmid encoding full-length MTA2 cDNA sequence and empty vector were purchased from Biogot Biotechnology (Nanjing, People's Republic of China). Plasmids were transfected into CNE1 cell by Lipofectamine 2000 (Thermo Fisher Scientific). Stable MTA2-overexpressing CNE1 cell (CNE1/MTA2) and negative control CNE1 cell (vector) were established under G418 (Sigma-Aldrich Co., St Louis, MO, USA) selection. The efficiency of MTA2 gene transfection was verified by qRT-PCR and Western blot.

\section{Establishment of stable MTA2-silencing CNE2 cell}

The expression of MTA2 in CNE2 cell was silenced by lentivirus-mediated short hairpin RNA (shRNA). The lentivirus particles containing shRNA targeting MTA2 and negative control shRNA were purchased from Genechem (Shanghai, People's Republic of China). For lentivirus infection, CNE2 cells were incubated with lentivirus and $5 \mu \mathrm{g} / \mathrm{mL}$ polybrene for 24 hours. After infection, cells were selected by flow cytometry with a green fluorescent protein marker. The efficiency of MTA2 knockdown was evaluated by qRT-PCR and Western blot. The stable MTA2-silencing and negative control CNE2 cells were named as CNE2/ shMTA2 and CNE2/shNC, respectively.

\section{CCK-8 proliferation assay}

Cell Counting Kit-8 (CCK-8) proliferation assay was performed as previously described. ${ }^{23}$ Briefly, cells were seeded in 96-well plates (2,000 cells per well) and grown in complete medium for 1 day, 2 days, 3 days, 4 days, or 5 days in a humidified atmosphere at $37^{\circ} \mathrm{C}$ with $5 \% \mathrm{CO}_{2}$. For analysis, $20 \mu \mathrm{L}$ of CCK-8 substrate was added to each well. After culturing for 2 hours, the absorbance of wells was measured with a microplate reader at $450 \mathrm{~nm}$.

\section{Colony formation assay}

Cells were seeded at a density of 100 cells per well in six-well plates. After culturing for 12 days in an incubator, the cells were fixed with methanol and stained with crystal violet. The number of colonies was counted under an inverse microscope.

\section{Wound healing assay}

Cells were seeded with the same number in six-well plates in complete medium. When cells grew to $\sim 95 \%$ confluence, scratch wounds were made with $100 \mu \mathrm{L}$ sterile pipette tips. To remove the suspended cells, the plates were washed with PBS twice. Cells were grown in DMEM containing 1\% FBS, and photos were taken in three defined fields at 0 hour and 48 hours, respectively. The closure area of wounds was measured with ImageJ software (National Institutes of Health, Bethesda, MD, USA).

\section{Transwell invasion assay}

Transwell cell culture inserts (pore size, $8 \mu \mathrm{m}$; Corning Incorporated, Corning, NY, USA) were coated with Matrigel (BD Biosciences, San Jose, CA, USA). Fifty thousand cells in $100 \mu \mathrm{L}$ serum-free DMEM were seeded in the upper chamber of the inserts. The bottom chamber was added with complete medium (DMEM containing 10\% FBS) as a chemoattractant. After incubation for 18 hours, cells in the upper surface of the membranes were removed with a cotton swab. Cells that had invaded to the lower surface of the membranes were fixed with methanol for 10 minutes and stained with $0.1 \%$ crystal violet for 30 minutes. Cells were observed under a microscope and counted in five predetermined fields.

\section{In vivo growth and metastasis experiments}

Four-week-old male BALB/c nu/nu mice were purchased from the Guangdong Medical Laboratory Animal Center and 
were bred in a specific pathogen-free condition. All animal study procedures were performed according to institutional guidelines.

For in vivo tumor growth experiment, the mice were injected subcutaneously in the right subaxillary with $100 \mu \mathrm{L}$ cell suspensions $\left(1 \times 10^{6}\right.$ cells $)$ of control or CNE2/shMTA2 cells ( $\mathrm{n}=6$ per group). Diameters of tumors were measured with a caliper weekly. The tumor volumes $(V)$ were calculated by the following formula: $V=a \times b^{2} / 2$ ( $a$ and $b$ represent the long and short axes of tumor, respectively). ${ }^{24}$ The mice were sacrificed after 3 weeks. Tumors were carefully removed and weighed.

For in vivo metastasis experiment, the mice were injected with $100 \mu \mathrm{L}$ cell suspensions $\left(1 \times 10^{6}\right.$ cells $)$ of control or CNE2/shMTA2 cells through the lateral tail vein $(n=6$ per group). After 6 weeks, the mice were sacrificed, and the lungs of mice were removed and subjected to hematoxylin and eosin staining. The number of pulmonary metastasis foci in each mouse was calculated as previously described..$^{25}$

\section{Statistical analysis}

Results are presented as mean \pm standard deviation of three independent experiments and analyzed by SPSS 13.0 software (SPSS Inc., Chicago, IL, USA). Chi-square test was performed for analysis of qualitative data. Student's $t$-test or one-way analysis of variance with least significant difference test for multiple comparisons was performed for analysis of quantitative data. $P<0.05$ was considered to be statistically significant.

\section{Results}

\section{MTA2 is upregulated in NPC and related to clinical features of patients}

We first investigated the expression of MTA2 in NPC tissues and cell lines. MTA2 protein was higher in NPC tissues $(60 / 107,56.1 \%)$ compared with noncancerous nasopharyngeal epithelium tissues $(8 / 28,28.6 \%)$, based on immunohistochemistry analysis. MTA2 protein is predominantly expressed in the nucleus of tumor cells (Figure 1A). Compared with nasopharyngeal epithelium cell line NP69, MTA2 protein levels were higher in NPC cell lines CNE1, CNE2, and HNE1 (Figure 1B). We further investigated the relationship between MTA2 expression and patient's clinicopathological features. High MTA2 expression was related to more advanced clinical stage and presence of lymph node metastasis but had no relation with patient's age, sex, and distant metastasis of tumor (Table 1).

\section{MTA2 overexpression enhances proliferation and migration of CNEI cell in vitro}

Among the three NPC cell lines detected, CNE1 cell had the lowest level of MTA2 protein and CNE2 cell had the highest level of MTA2 protein (Figure 1B). For this reason,
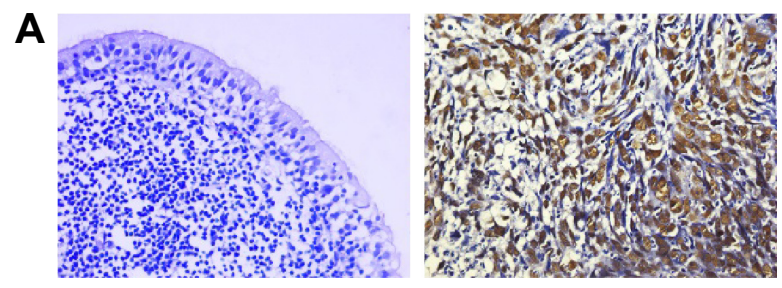

B
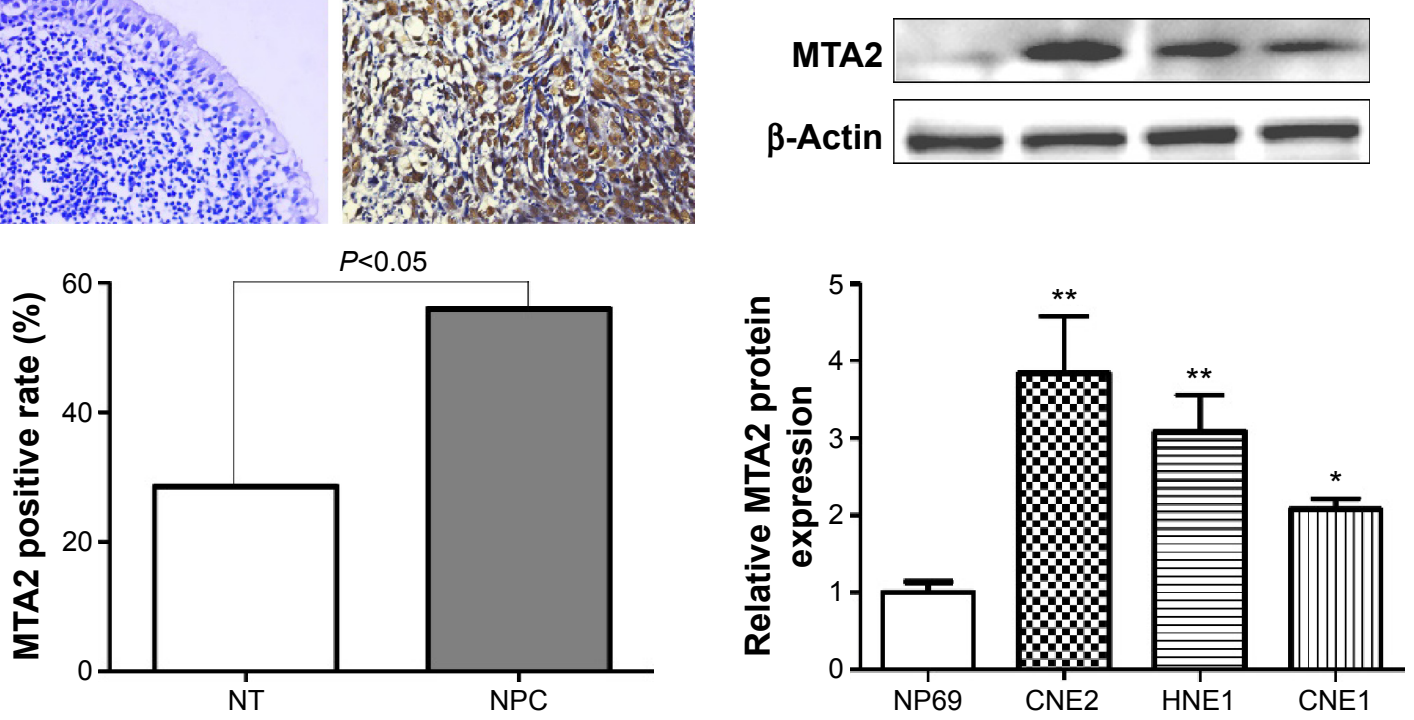

Figure I MTA2 is upregulated in NPC.

Notes: (A) Representative negative immunostaining in a noncancerous nasopharyngeal epithelium (NT) tissue and positive nuclear immunostaining in a NPC tissue Magnification: $\times 400$. MTA2 expression is higher in NPC tissues compared with noncancerous tissues. (B) MTA2 protein expression in CNEI, CNE2, HNEI NPC cell lines and NT NP69 cells detected by Western blot (**P<0.01 vs NP69, $* P<0.05$ vs NP69).

Abbreviations: MTA2, metastasis-associated gene 2; NPC, nasopharyngeal carcinoma. 
Table I Relationship between MTA2 expression and clinicopathological features of patients with NPC

\begin{tabular}{|c|c|c|c|c|c|}
\hline \multirow[t]{2}{*}{ Variables } & \multirow[t]{2}{*}{$\mathbf{n}$} & \multicolumn{2}{|c|}{ MTA2 expression } & \multirow[t]{2}{*}{$\chi^{2}$} & \multirow[t]{2}{*}{$P$-value } \\
\hline & & $-(\%)$ & $+(\%)$ & & \\
\hline \multicolumn{6}{|l|}{ Sex } \\
\hline Male & 71 & 34 (47.9) & $37(52.1)$ & 1.345 & 0.246 \\
\hline Female & 36 & $13(36.1)$ & $23(63.9)$ & & \\
\hline \multicolumn{6}{|l|}{ Age (years) } \\
\hline$<50$ & 60 & $29(48.3)$ & $31(5 \mid .7)$ & 1.078 & 0.299 \\
\hline$\geq 50$ & 47 & $18(38.3)$ & $29(61.7)$ & & \\
\hline \multicolumn{6}{|l|}{ Clinical stage } \\
\hline Stages I-II & 18 & $13(72.2)$ & $5(27.8)$ & 7.035 & $<0.01$ \\
\hline Stages III-IV & 89 & $34(38.2)$ & $55(61.8)$ & & \\
\hline \multicolumn{6}{|c|}{ Lymph node metastasis } \\
\hline No & 28 & $19(67.9)$ & $9(32.1)$ & 8.818 & $<0.01$ \\
\hline Yes & 79 & $28(35.4)$ & $51(64.6)$ & & \\
\hline \multicolumn{6}{|c|}{ Distant metastasis } \\
\hline No & 94 & $43(45.7)$ & $51(54.3)$ & 1.040 & 0.308 \\
\hline Yes & 13 & $4(30.8)$ & $9(69.2)$ & & \\
\hline
\end{tabular}

Abbreviations: MTA2, metastasis-associated gene 2; NPC, nasopharyngeal carcinoma.

CNE1 and CNE2 cells were chosen for the gain-of-function and loss-of-function studies, respectively. Stable MTA2overexpressing CNE1 cell (CNE1/MTA2) and negative control (vector) were established by plasmid transfection. Stable MTA2-silencing CNE2 cell (shMTA2) and negative control (shNC) were established by lentivirus-mediated shRNA.

We found a 25-fold increase in MTA2 mRNA level and a remarkable upregulation in MTA2 protein level in CNE1/ MTA2 cell compared with control (Figure 2A). CNE1/ MTA2 cell had a higher proliferation rate in CCK-8 assay and formed more colonies in colony formation assay as compared with control (Figure 2B and C). The influence of MTA2 upregulation on migration and invasion of NPC cell was investigated by wound healing assay and transwell assay. Compared with control, CNE1/MTA2 cell had a higher wound closure rate (Figure 2D). Transwell invasion assay also showed a significant increase in the number of invaded cells in CNE1/MTA2 cell (Figure 2E).

\section{MTA2 depletion suppresses migration and invasion of CNE2 cell in vitro}

MTA2 expression was successfully attenuated by lentivirusmediated shRNA determined by qRT-PCR and Western blot (Figure 3A). MTA2 depletion inhibited proliferation of CNE2 cells by CCK-8 and colony formation assay (Figure 3B and C). MTA2 depletion also impaired the migration and invasion ability of CNE2 cell, which was identified by wound healing and transwell assay (Figure 3D and E).

\section{MTA2 activates the Akt signaling pathway in NPC}

We investigated whether the Akt signaling pathway was involved in the function of MTA2. We detected the protein levels of total Akt, activated Akt (pAkt), and some downstream genes closely related to cell growth and invasion. Compared with control cells, MTA2 overexpression induced upregulation of pAkt and cyclin D1 and MMP7 in CNE1/MTA2 cell (Figure 4A), while MTA2 knockdown caused downregulation of these genes in CNE2/shMTA2 cell (Figure 4B).

\section{MTA2 depletion inhibits growth, metastasis, and Akt activity of CNE2 cell in vivo}

We further explored the influence of MTA2 knockdown on growth and metastasis of CNE2 cell in vivo. Both control and CNE2/shMTA2 groups formed tumors in nude mice after injection of tumor cells subcutaneously, but the tumor weight in CNE2/shMTA2 group was only $49 \%$ of the control group (Figure 5A). The tumor volume was also smaller in CNE2/shMTA2 group (data not shown). Furthermore, CNE2/ shMTA2 group formed much fewer pulmonary metastatic foci compared with control group after injection of tumor cells through tail vein (Figure 5B). Finally, the expression of MTA2, pAkt, cyclin D1, and MMP7 proteins in subcutaneous tumors of CNE2/shMTA2 group were lower than the control group (Figure 5C).

\section{Discussion}

Although MTA2 is reported to be overexpressed in several human malignancies and involved in the progression of tumor, ${ }^{13}$ little is known about the role of MTA2 in NPC. For the first time, we identified the upregulation of MTA2 in NPC tissues. Moreover, we also confirmed an overexpression of MTA2 in three NPC cell lines detected compared with nasopharyngeal epithelium cell line NP69. We concluded that MTA2 was upregulated in NPC and may participate in the carcinogenesis of NPC.

In NPC tissues, MTA2 protein appeared in the nucleus of tumor cells and no obvious cytoplasmic staining was observed, which is consistent with previous studies. ${ }^{26}$ MTA2 expression was positively related to clinical stage and lymph node metastasis of patients, indicating that MTA2 might be indispensable in the progression of NPC.

In the three NPC cell lines detected, CNE2 cell had the highest and CNE1 cell had the lowest level of MTA2 protein. 
A
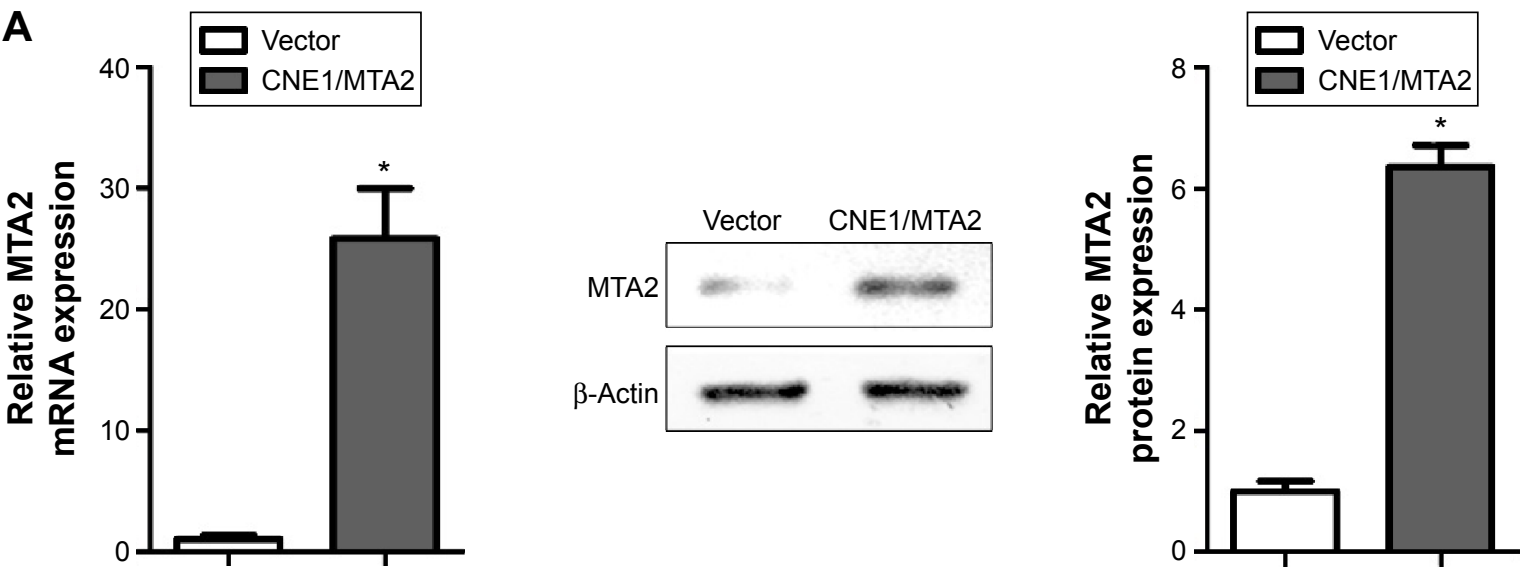

B

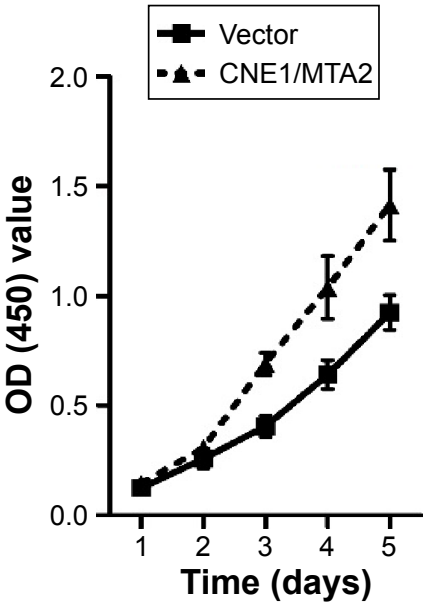

D

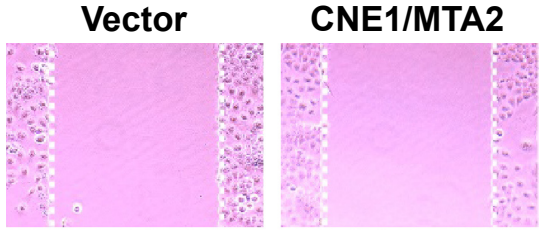

$\mathrm{Oh}$
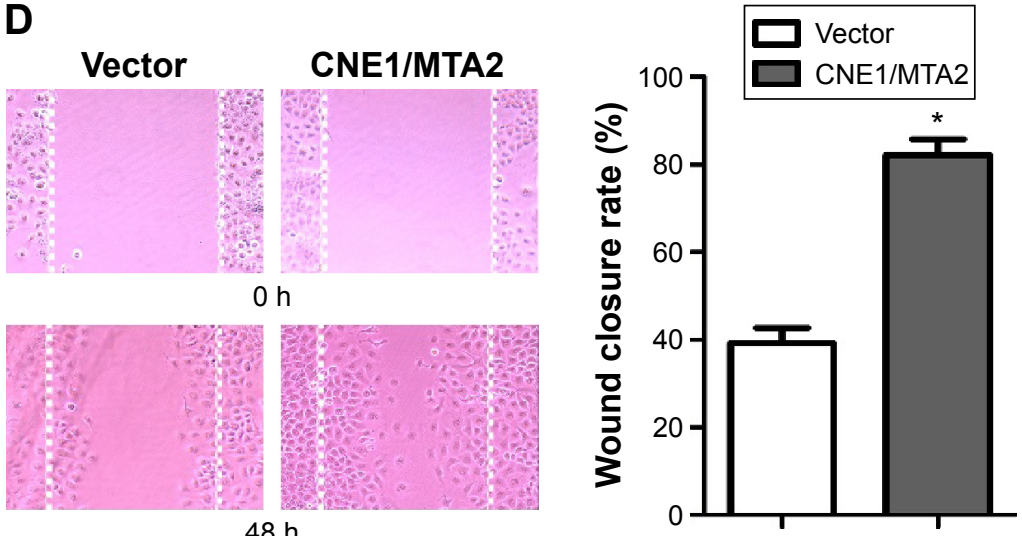

C

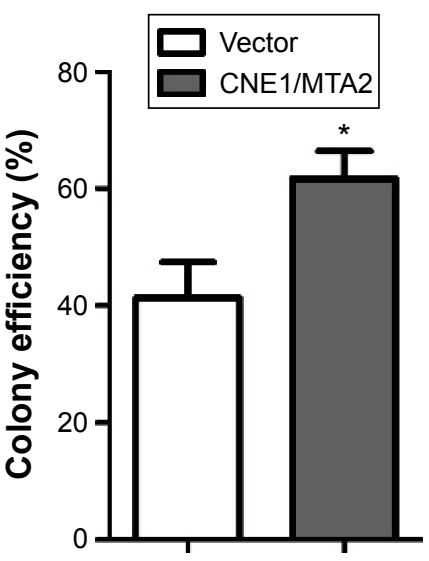

E
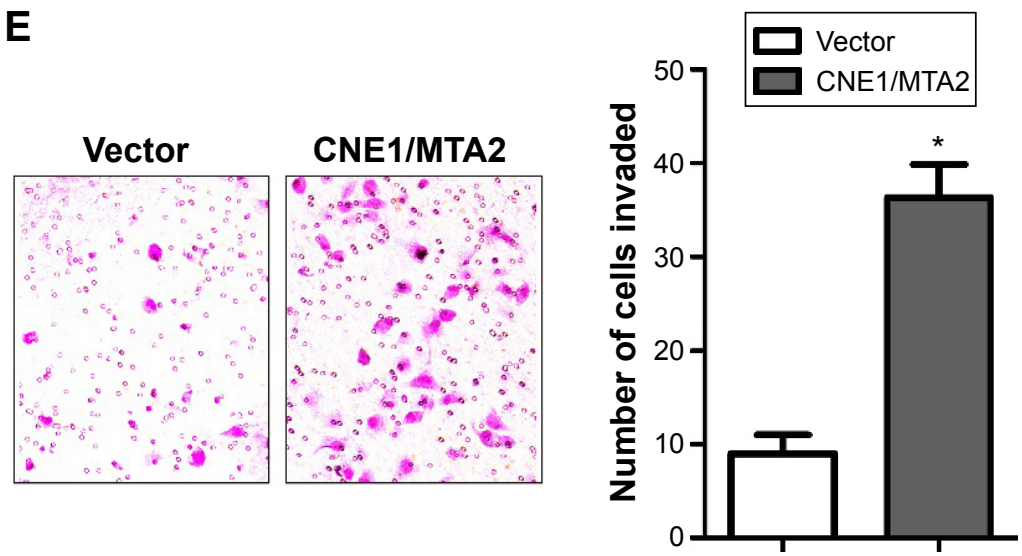

Figure 2 MTA2 overexpression promotes proliferation and migration of CNEI cells.

Notes: (A) MTA2 mRNA and protein levels of control and CNEI/MTA2 cells detected by qRT-PCR and Western blot. (B and C) Detection of cell viability by CCK-8 and colony formation assay. Detection of cell migration and invasion capability by wound healing $([\mathrm{D}]$ magnification: $\times 100)$ and transwell invasion assay $([\mathrm{E}]$ magnification: $\times 200) ;(* P<0.0 \mathrm{I})$. Abbreviations: MTA2, metastasis-associated gene 2; mRNA, messenger RNA; OD, optical density; qRT-PCR, quantitative real-time reverse transcription-polymerase chain reaction; CCK-8, Cell Counting Kit-8; h, hours.

In order to perform functional study, MTA2 was upregulated in CNE1 cell and downregulated in CNE2 cell. We found that ectopic overexpression of MTA2 in CNE1 cell improved cell viability and colony efficiency as well as cell migration and invasion ability. On the other hand, MTA2 depletion in CNE2 cell produced opposite effects. The xenograft experiments verified that MTA2 depletion attenuated cell growth and metastasis in vivo. The results highlighted the importance of MTA2 in proliferation, migration, and invasion of NPC cells. 
A
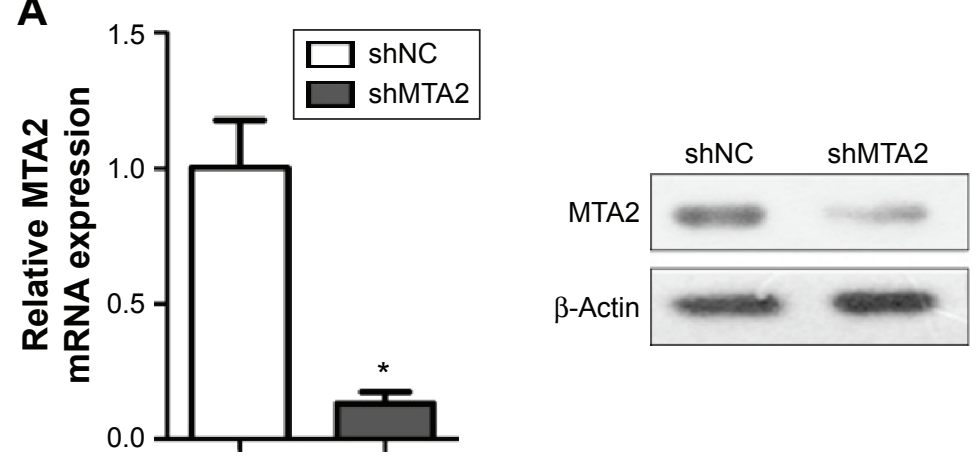

B

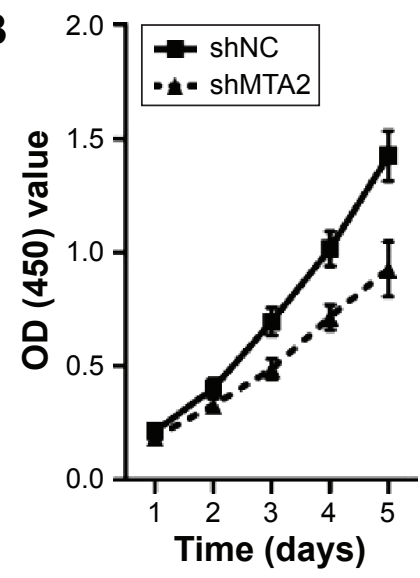

C

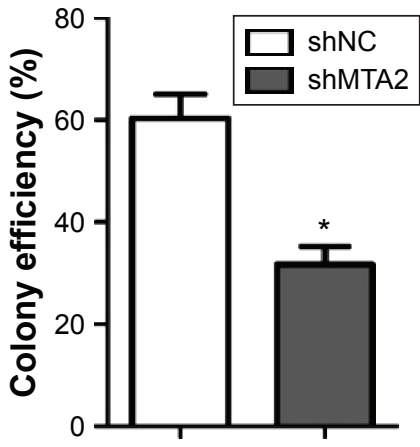

D

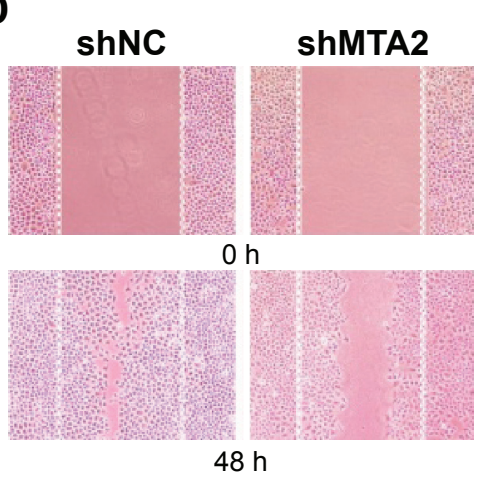

$E$

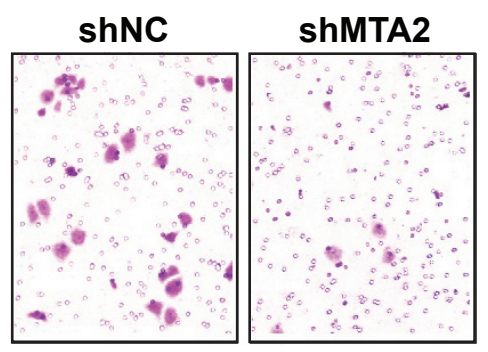

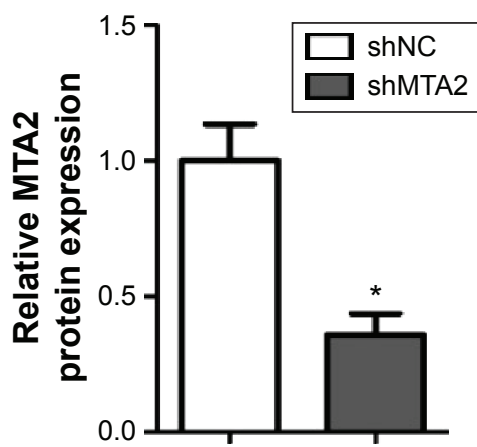
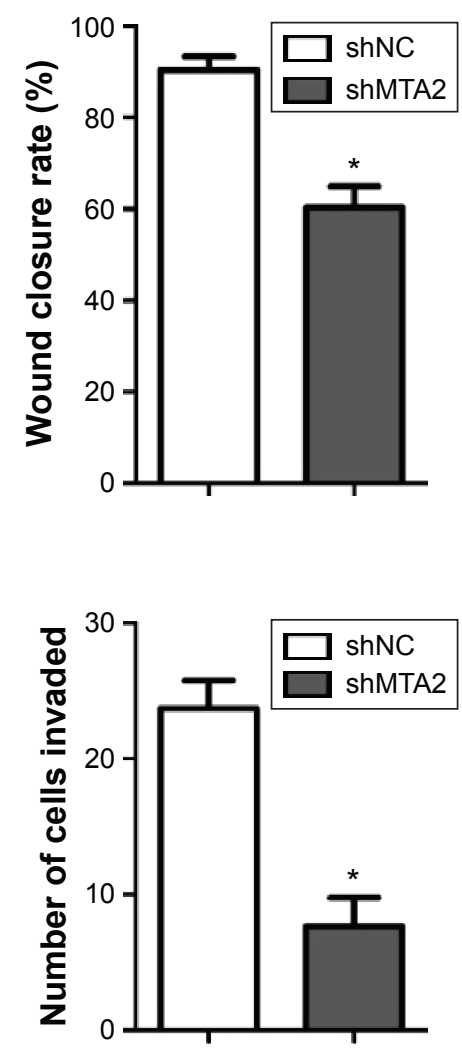

Figure 3 MTA2 knockdown inhibits proliferation and migration of CNE2 cells.

Notes: (A) Analysis of MTA2 expression by qRT-PCR and Western blot in CNE2/shNC and CNE2/shMTA2 cells. (B and C) Analysis of cell viability and colony efficiency by CCK-8 and colony formation assay. (D and $\mathbf{E})$ Analysis of cell migration and invasion ability by wound healing $([\mathbf{D}]$ magnification: $\times 100)$ and transwell invasion assay $([\mathbf{E}]$ magnification: $\times 200) ;(* P<0.01)$.

Abbreviations: MTA2, metastasis-associated gene 2; OD, optical density; qRT-PCR, quantitative real-time reverse transcription-polymerase chain reaction; mRNA, messenger RNA; CCK-8, Cell Counting Kit-8; h, hours.

MTA2 had been reported to influence tumor cell growth and migration. For example, ectopic expression of MTA2 promoted tumor cell growth and migration in gastric cancer and stimulated interleukin 11 secretion. ${ }^{27}$ In human breast cancer, MTA2 overexpression promoted cell invasion by activating RhoA pathway. ${ }^{28}$ MTA2 depletion affected the expression of apoptosis-related genes $P 21, P 27, P 53$, and Bax and metastasis-related genes MMP2 and MMP9. ${ }^{29}$ MTA2 had been proved to influence cell apoptosis. ${ }^{30}$ The impact of MTA2 on apoptosis of NPC cell is worthy of further investigation.
The PI3K/Akt signaling pathway plays an important role in cell proliferation, differentiation, and migration. ${ }^{31,32}$ Several studies indicated a close relationship between MTA1 (a member of MTA family with a structure similar to MTA2) and Akt signaling pathway. ${ }^{33,34}$ Moreover, silencing of MTA2 changed the expression of some downstream genes of Akt pathway such as $P 21$ and $P 53,{ }^{15,29}$ suggesting a possible interaction between MTA2 and Akt pathway. Therefore, we compared the protein expression of Akt, pAkt, and some downstream genes such as cyclin D1 and MMP7 in NPC cells. Akt was activated by MTA2 upregulation and suppressed 

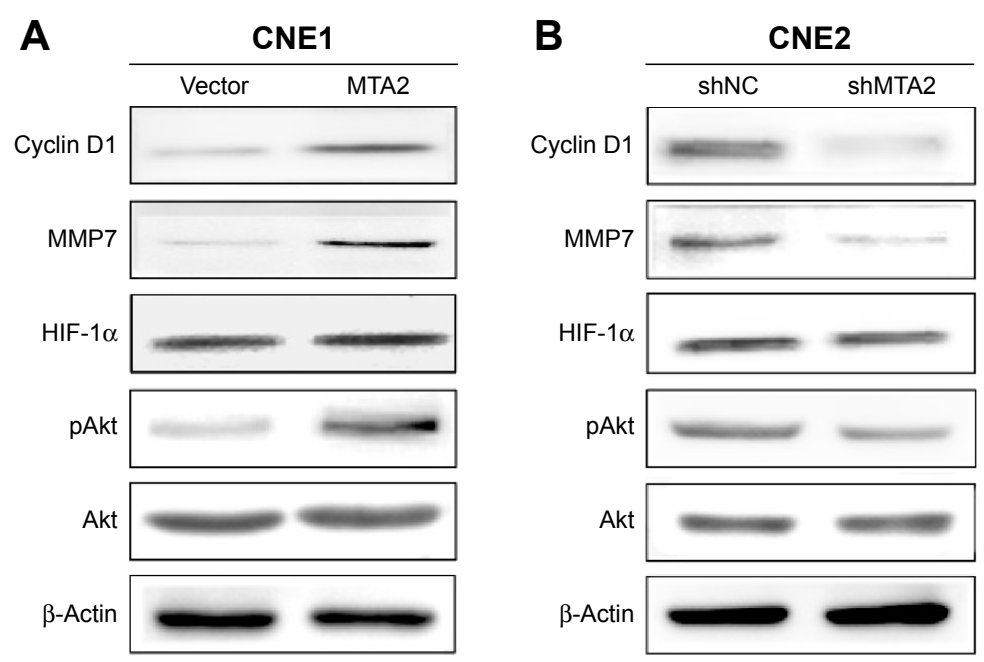

Figure 4 MTA2 stimulates Akt signaling pathway in NPC cells.

Notes: Western blot analysis of Akt, pAkt, HIF-I $\alpha$, MMP7, and cyclin DI proteins expression in CNEI/vector and CNEI/MTA2 cells (A) or CNEI/shNC and CNE2/shMTA2 cells (B).

Abbreviations: MTA2, metastasis-associated gene 2; NPC, nasopharyngeal carcinoma; HIF-I $\alpha$, hypoxia-inducible factor I $\alpha$; MMP7, matrix metalloproteinase 7.

A

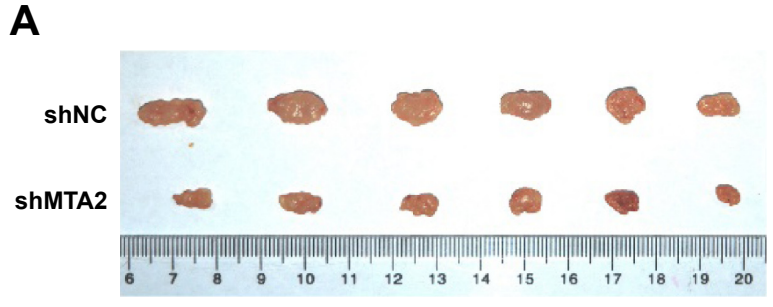

B

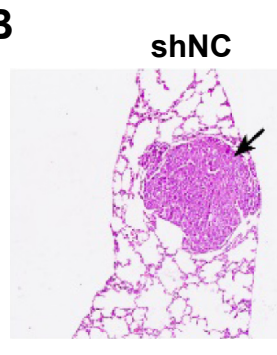

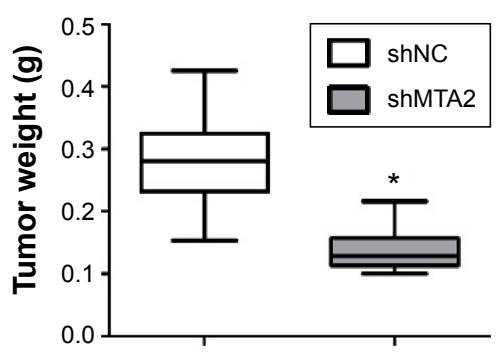

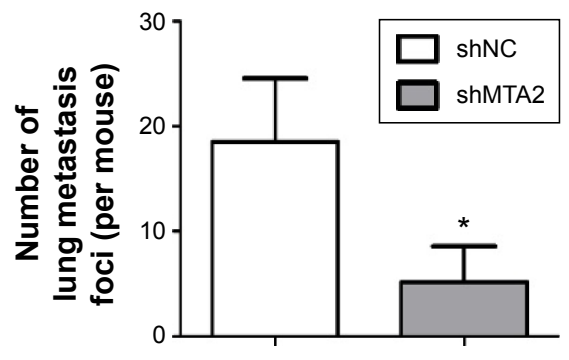

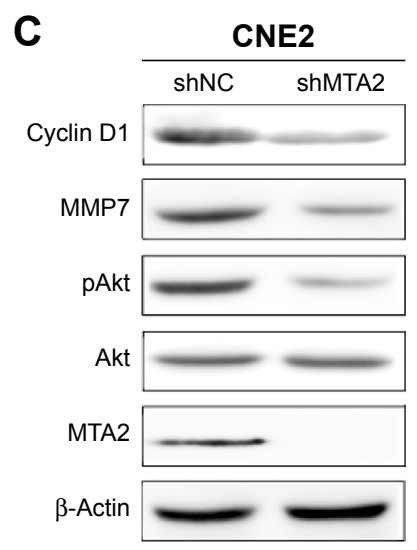

Figure 5 Knockdown of MTA2 suppresses growth and metastasis of CNE2 cells in vivo.

Notes: (A) Tumor size and tumor weight of CNE2/shNC and CNE2/shMTA2 groups in nude mice 3 weeks after injection of control or CNE2/shMTA2 cells subcutaneously in the right subaxillary. (B) Observation of pulmonary metastasis foci (black arrow) of nude mice 6 weeks after injection of control or CNE2/shMTA2 cells through tail vein (H\&E $\times 100, * P<0.01)$. (C) Levels of MTA2, pAkt, cyclin DI, and MMP7 proteins in subcutaneous tumors of CNE2/shNC and CNE2/shMTA2 groups.

Abbreviations: MTA2, metastasis-associated gene 2; H\&E, hematoxylin and eosin; MMP7, matrix metalloproteinase 7. 
by MTA2 depletion, as well as protein levels of MMP7 and cyclin D1. Nude mice experiment further verifies that MTA2 depletion inhibits Akt activity in vivo, indicating that MTA2 might regulate expression of MMP7 and cyclin D1 via Akt pathway in NPC cells.

\section{Conclusion}

In summary, we find that MTA2 is overexpressed in NPC tissues and cell lines. Upregulation of MTA2 promotes proliferation and invasion of NPC cells, while downregulation of MTA2 impairs cell growth and invasion, both in vitro and in vivo. Furthermore, MTA2 silencing inhibits Akt pathway. This work strongly suggests that MTA2 plays an important role in the development of NPC and may be a potential therapy target.

\section{Acknowledgments}

This work is supported by the National Natural Science Foundation of China (81171824, 81371719, and 81371509), Colleges Pearl River Scholar Funded Scheme (GDUPS, 2013), the Foundation for Distinguished Young Teachers Plan in Higher Education of Guangdong Province (YQ2013087), and the Research foundation of Guangdong Medical University (M2012002, M2015011).

\section{Disclosure}

The authors report no conflicts of interest in this work.

\section{References}

1. Wei KR, Zheng RS, Zhang SW, Liang ZH, Ou ZX, Chen WQ. Nasopharyngeal carcinoma incidence and mortality in China in 2010. Chin J Cancer. 2014;33(8):381-387.

2. Huang TR, Zhang SW, Chen WQ, et al. Trends in nasopharyngeal carcinoma mortality in China, 1973-2005. Asian Pac J Cancer Prev. 2012;13(6):2495-2502.

3. Millard CJ, Fairall L, Schwabe JW. Towards an understanding of the structure and function of MTA1. Cancer Metastasis Rev. 2014;33(4): 857-867.

4. Sen N, Gui B, Kumar R. Physiological functions of MTA family of proteins. Cancer Metastasis Rev. 2014;33(4):869-877.

5. Wu M, Wang L, Li Q, Li J, Qin J, Wong J. The MTA family proteins as novel histone $\mathrm{H} 3$ binding proteins. Cell Biosci. 2013;3(1):1.

6. Li DQ, Yang Y, Kumar R. MTA family of proteins in DNA damage response: mechanistic insights and potential applications. Cancer Metastasis Rev. 2014;33(4):993-1000.

7. Park HR, Jung WW, Kim HS, Bacchini P, Bertoni F, Park YK. Overexpression of metastatic tumor antigen in osteosarcoma: comparison between conventional high-grade and central low-grade osteosarcoma. Cancer Res Treat. 2005;37(6):360-364.

8. Ding W, Hu W, Yang H, Ying T, Tian Y. Prognostic correlation between MTA2 expression level and colorectal cancer. Int J Clin Exp Pathol. 2015; 8(6):7173-7180.

9. Ji Y, Zhang P, Lu Y, Ma D. Expression of MTA2 gene in ovarian epithelial cancer and its clinical implication. J Huazhong Univ Sci Technolog Med Sci. 2006;26(3):359-362.
10. Zhang B, Zhang H, Shen G. Metastasis-associated protein 2 (MTA2) promotes the metastasis of non-small-cell lung cancer through the inhibition of the cell adhesion molecule Ep-CAM and E-cadherin. Jpn J Clin Oncol. 2015;45(8):755-766.

11. Liu YP, Shan BE, Wang XL, Ma L. Correlation between MTA2 overexpression and tumour progression in esophageal squamous cell carcinoma. Exp Ther Med. 2012;3(4):745-749.

12. Chen DW, Fan YF, Li J, Jiang XX. MTA2 expression is a novel prognostic marker for pancreatic ductal adenocarcinoma. Tumour Biol. 2013; 34(3):1553-1557.

13. Covington KR, Fuqua SA. Role of MTA2 in human cancer. Cancer Metastasis Rev. 2014;33(4):921-928.

14. Kumar R, Wang RA, Mazumdar A, et al. A naturally occurring MTA1 variant sequesters oestrogen receptor-alpha in the cytoplasm. Nature. 2002;418(6898):654-657.

15. Cheng CY, Chou YE, Ko CP, et al. Metastasis tumor-associated protein-2 knockdown suppresses the proliferation and invasion of human glioma cells in vitro and in vivo. $J$ Neurooncol. 2014;120(2):273-281.

16. Zhou C, Ji J, Cai Q, et al. MTA2 promotes gastric cancer cells invasion and is transcriptionally regulated by Sp1. Mol Cancer. 2013;12:102.

17. Fu J, Qin L, He T, et al. The TWIST/Mi2/NuRD protein complex and its essential role in cancer metastasis. Cell Res. 2011;21(2):275-289.

18. Soumaoro LT, Uetake H, Higuchi T, et al. Cyclooxygenase-2 expression: a significant prognostic indicator for patients with colorectal cancer. Clin Cancer Res. 2004;10(24):8465-8471.

19. Tsao SW, Wang X, Liu Y, et al. Establishment of two immortalized nasopharyngeal epithelial cell lines using SV40 large T and HPV16E6/E7 viral oncogenes. Biochim Biophys Acta. 2002;1590(1-3):150-158.

20. Ma L, Deng X, Wu M, Zhang G, Huang J. Down-regulation of miRNA-204 by LMP-1 enhances CDC42 activity and facilitates invasion of EBV-associated nasopharyngeal carcinoma cells. FEBS Lett. 2014;588(9):1562-1570.

21. Livak KJ, Schmittgen TD. Analysis of relative gene expression data using real-time quantitative PCR and the 2(-Delta Delta $\mathrm{C}(\mathrm{T})$ ) Method. Methods. 2001;25(4):402-408.

22. Deng X, Ma L, Wu M, et al. miR-124 radiosensitizes human glioma cells by targeting CDK4. J Neurooncol. 2013;114(3):263-274.

23. Shen Z, Zeng Y, Guo J, et al. Over-expression of the special AT rich sequence binding protein 1 (SATB1) promotes the progression of nasopharyngeal carcinoma: association with EBV LMP-1 expression. J Transl Med. 2013;11:217.

24. Caysa H, Hoffmann S, Luetzkendorf J, et al. Monitoring of xenograft tumor growth and response to chemotherapy by non-invasive in vivo multispectral fluorescence imaging. PLoS One. 2012;7(10):e47927.

25. Jiang $\mathrm{H}$, Gao M, Shen $\mathrm{Z}$, et al. Blocking PI3K/Akt signaling attenuates metastasis of nasopharyngeal carcinoma cells through induction of mesenchymal-epithelial reverting transition. Oncol Rep. 2014;32(2): $559-566$.

26. Liu J, Wang H, Huang C, Qian H. Subcellular localization of MTA proteins in normal and cancer cells. Cancer Metastasis Rev. 2014;33(4): $843-856$.

27. Zhou C, Ji J, Cai Q, et al. MTA2 enhances colony formation and tumor growth of gastric cancer cells through IL-11. BMC Cancer. 2015;15:343.

28. Covington KR, Brusco L, Barone I, et al. Metastasis tumor-associated protein 2 enhances metastatic behavior and is associated with poor outcomes in estrogen receptor-negative breast cancer. Breast Cancer Res Treat. 2013;141(3):375-384.

29. Lu J, Jin ML. Short-hairpin RNA-mediated MTA2 silencing inhibits human breast cancer cell line MDA-MB231 proliferation and metastasis. Asian Pac J Cancer Prev. 2014;15(14):5577-5582.

30. Luo J, Su F, Chen D, Shiloh A, Gu W. Deacetylation of p53 modulates its effect on cell growth and apoptosis. Nature. 2000;408(6810): $377-381$.

31. Nicholson KM, Anderson NG. The protein kinase B/Akt signalling pathway in human malignancy. Cell Signal. 2002;14(5):381-395.

32. Ying J, Xu Q, Liu B, Zhang G, Chen L, Pan H. The expression of the $\mathrm{PI} 3 \mathrm{~K} / \mathrm{AKT} / \mathrm{mTOR}$ pathway in gastric cancer and its role in gastric cancer prognosis. Onco Targets Ther. 2015;8:2427-2433. 
33. Wang H, Fan L, Wei J, et al. Akt mediates metastasis-associated gene 1 (MTA1) regulating the expression of E-cadherin and promoting the invasiveness of prostate cancer cells. PLoS One. 2012;7(12):e46888.
34. Feng X, Zhang Q, Xia S, et al. MTA1 overexpression induces cisplatin resistance innasopharyngeal carcinoma by promoting cancer stem cells properties. Mol Cells. 2014;37(9):699-704.

\section{Publish your work in this journal}

OncoTargets and Therapy is an international, peer-reviewed, open access journal focusing on the pathological basis of all cancers, potential targets for therapy and treatment protocols employed to improve the management of cancer patients. The journal also focuses on the impact of management programs and new therapeutic agents and protocols on
Dovepress

patient perspectives such as quality of life, adherence and satisfaction The manuscript management system is completely online and includes a very quick and fair peer-review system, which is all easy to use. Visit http://www.dovepress.com/testimonials.php to read real quotes from published authors. 